\title{
Carcinoma epidermoide em mucosa bucal: um breve levantamento
}

\author{
Squamous cell carcinoma in mouth mucosa: a short survey
}

Fernando Augusto Cervantes Garcia De Sousa ${ }^{1}$, Thaís Cachuté Paradella ${ }^{2}$, Luiz Eduardo Blumer Rosa ${ }^{3}$, Horácio Faig-Leite ${ }^{4}$

\section{Resumo}

O câncer bucal é um problema de saúde pública em muitos países, inclusive no Brasil. Todavia, a prevenção e o diagnóstico precoce continuam sendo as formas mais eficazes de combate ao câncer.

Traçar um perfil dos casos de carcinoma epidermoide na cidade de São José dos Campos, SP.

Foram levantados todos os laudos emitidos no período de 1972 a 2004, sendo que desses, apenas 224 continham o diagnóstico de interesse. A seguir, foram coletados os dados referentes ao sexo, à raça, à idade e à localização da lesão primária. Estes foram, então, tabulados e analisados, utilizando-se da estatística descritiva.

Quanto ao sexo, observou-se uma forte predileção pelo masculino $(80,4 \%)$, o mesmo acontecendo em relação à raça branca $(74,6 \%)$. Já a respeito da idade dos pacientes no momento do diagnóstico, $86,2 \%$ estavam entre a quinta e a oitava década de vida. Neste estudo, também se pode notar que a língua foi a região mais acometida, com $22,8 \%$ dos casos, seguida do lábio inferior, $19,7 \%$, e do soalho bucal, $14,3 \%$.

O padrão epidemiológico apresentado pelo câncer bucal na cidade de São José dos Campos, SP e região não difere daquele observado no restante do Brasil.

Palavras-chave: Câncer bucal, Carcinoma epidermóide, Epidemiologia

\section{Abstract}

Oral cancer is a public health issue in many countries, including Brazil. Thus, prevention and early diagnosis still remain as the most effective ways to combat cancer.

The present study aimed to obtain a profile of squamous cell carcinoma cases in the city of São José dos Campos.

All case files from 1972 to 2004 were analyzed and only 224 had the diagnosis of interest. Data regarding gender, race, age and location of the primary lesion were tabled and analyzed, using descriptive statistics.

Regarding gender, a strong occurrence in males $(80.4 \%$ of the cases) was observed, the same regarding white race $(74.6 \%)$. In regards to age, $86.2 \%$ of the patients were between the fifth and eighth decade of life at the moment of the diagnosis. In this study, is was also noticeable that tongue was the most affected region, with $22.8 \%$ of the cases, followed by inferior lip (19.7\%) and mouth floor $(14.3 \%)$.

The epidemiological pattern presented by oral cancer in the city of São José dos Campos does not differ from the one observed in the other parts of Brazil.

Keywords: Mouth neoplasms, Squamous cell carcinoma, Epidemology
${ }^{1}$ Doutor em Biopatologia Bucal pelo Programa de Pós-Graduação em Biopatologia Bucal da Faculdade de Odontologia de São José dos Campos - UNESP

2 Doutora em Biopatologia Bucal pelo Programa de Pós-Graduação em Biopatologia Bucal da Faculdade de Odontologia de São José dos Campos - UNESP

${ }^{3}$ Professor-Adjunto da Disciplina de Patologia Bucal da Faculdade de Odontologia de São José dos Campos - UNESP

${ }^{4}$ Professor Titular da Disciplina de Anatomia da Faculdade de Odontologia de São José dos Campos - UNESP

Correspondência: Dr. Fernando Augusto Cervantes Garcia de Souza

Endereço: R. Irmã Demétria Kfuri, 196 - Jardim Esplanada II - CEP 12242-500

São José dos Campos - SP, Brasil

Fone: 1239239080 - Fax: 1239479010

E-mail: facgs@uol.com.br

\section{Introdução}

Segundo estimativas do Instituto Nacional do Câncer (INCA) para o ano de 2005, serão diagnosticados no Brasil 467.440 novos casos de câncer, sendo que, desses, 13.880 acometerão a cavidade bucal, colocando-a como o sexto local de maior incidência da doença entre os homens e a oitava entre as mulheres. Estes números mostram que, apesar de todos os avanços obtidos, o câncer bucal continua sendo um problema de saúde pública (BRASIL, 2004).

Cabe, portanto, as autoridades competentes estabelecerem programas que incentivem a prevenção e o diagnóstico precoce desta doença. Considerando principalmente que, a exemplo do que ocorre com as demais neoplasias malignas, o câncer bucal tem a sua etiologia fortemente associada a fatores ambientais, possibilitando, assim, a adoção de medidas preventivas bastante eficazes. Entre esses fatores, o tabagismo é o que merece maior destaque (PARISE JUNIOR, 2000). Diversos estudos sugerem que o consumo de tabaco aumenta consideravelmente o risco de desenvolvimento da doença (FRANCO et al., 1989; VIGNESWARAN et al., 1995), em especial, quando associado ao etilismo (MÖLLER, 1989; BOYLE et al., 1990; KO et al., 1995). No entanto, vale ressaltar que ambos podem ser evitados, desde que se consiga conscientizar o indivíduo dos seus malefícios.

Além disso, mesmo que não se possa atuar antes do aparecimento das primeiras alterações clínicas, o diagnóstico precoce possibilita uma evolução favorável da doença. Isto porque mais de $90 \%$ dos casos de câncer bucal corresponde ao carcinoma epidermoide, cujo potencial de metástase nas fases iniciais é baixo (PARISE JUNIOR, 2000; NEVILLE et al., 2004). Infelizmente, $80 \%$ dos pacientes que procuram ou são encaminhados para hospitais e centros especializados de diagnóstico e tratamento do câncer bucal já se encontram em estágios avançados da doença, fase em que as possibilidades de cura estão drasticamente reduzidas, alcançando uma taxa de mortalidade superior a $60 \%$ (BRASIL, 1996).

Contudo, antes de se estabelecer qualquer estratégia de combate ao câncer, é necessário conhecer o perfil dos pacientes acometidos pela doença, permitindo identificar aqueles indivíduos de maior risco. Neste contexto, o presente trabalho tem como objetivo descrever as características epidemiológicas dos casos de carcinoma epidermóide diagnosticados na cidade de com outros levantamentos realizados no Brasil nos últimos anos. 


\section{Material e Métodos}

Foram avaliados todos os laudos com o diagnóstico histopatológico de carcinoma epidermoide em mucosa bucal emitidos pelo Serviço de Patologia Cirúrgica da Faculdade de Odontologia de São José dos Campos, UNESP, entre 1972 e 2004. A seguir, foram selecionados os dados referentes ao sexo, à raça (branca, negra e amarela), e à idade dos pacientes. Além da localização da lesão primária.

Quanto à localização, foram padronizados os seguintes sítios anatômicos: área retromolar, gengiva, lábio, língua, mucosa jugal, palato, rebordo alveolar e soalho bucal. Os casos nos quais a localização não foi mencionada ou houve alguma dúvida foram agrupados como informação não-especificada. Este critério também foi utilizado para os dados acerca da raça e da idade. descritivamente.

Os dados obtidos foram, então, tabulados e analisados

\section{Resultado e Discussão}

Entre 1972 e 2004, foram diagnosticados 224 casos de carcinoma epidermoide na mucosa bucal, com uma média de 6,8 casos por ano. No entanto, observa-se que, a partir de 1993 , esta média subiu para 14,8 , o que mostra um aumento considerável em relação ao período de 1972 a 1992, cuja média manteve-se inferior a 3 casos por ano.

Em termos de incidência, o ano de 2003 foi o que mais se sobressaiu, com 34 casos diagnosticados, contrapondo-se aos anos de 1974, 1977, 1980 e 1984, nas quais não foram diagnosticados nenhum novo caso (Gráfico 1).

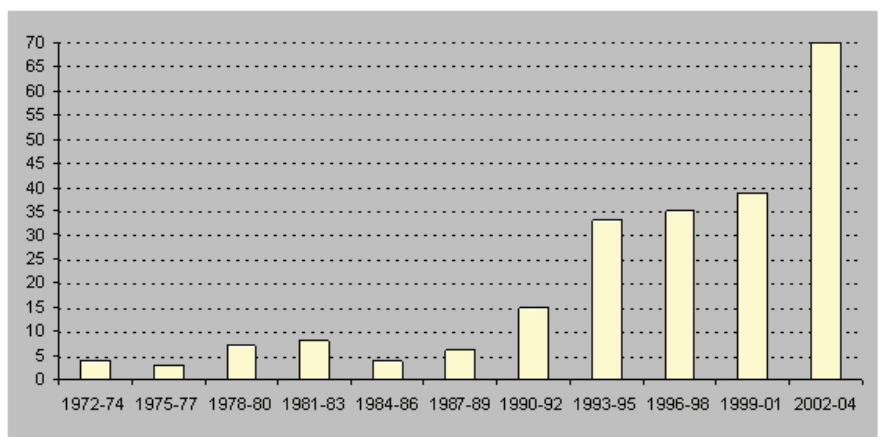

Gráfico 1. Distribuição do número de casos diagnosticados por triênio.

Vários fatores podem estar relacionados com 0 acréscimo do número de caso após 1993. Contudo, a consolidação da cidade de São José dos Campos como referência regional no diagnóstico do câncer bucal após a década de 90 parece ser o de maior relevância. Outro fator que merece atenção é o aumento da importância dada à prevenção e ao diagnóstico precoce, tanto pelo profissional quanto pelo paciente, levando a uma busca cada vez maior dos hospitais e centros especializados.

Quanto ao sexo, os homens responderam a $80,4 \%$ dos casos, corroborando com a literatura (CAMARINI, 1999; FIGUEIREDO et al., 2000; ABDO, 2001; ANJOS HORA et al., 2003). No entanto, $86,4 \%$ dos casos que acometeram o sexo feminino ocorreram a partir dos anos 90 , o que pode ser explicado por uma maior difusão do hábito de fumar entre as muIheres nos últimos anos. Esta tendência é facilmente observada no câncer de pulmão, cuja taxa de mortalidade, desde 1979 até 1999 , cresceu $57 \%$ no sexo masculino e $122 \%$ no feminino (BRASIL, 2002) (Gráfico 2).

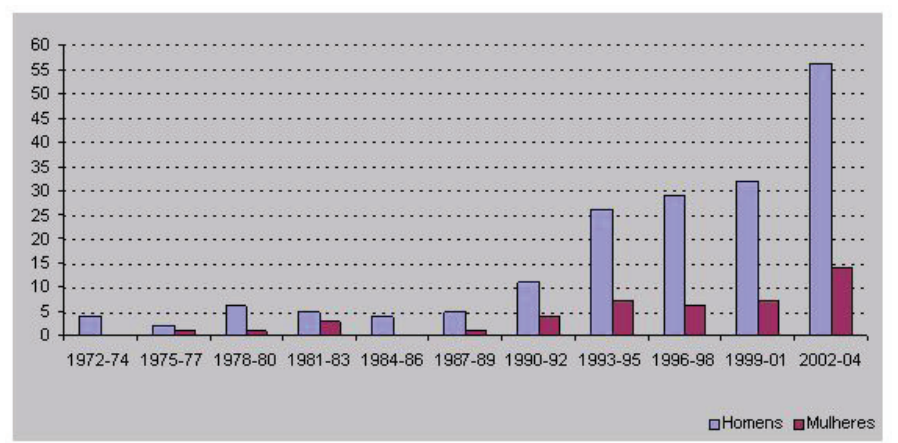

Gráfico 2 . Número de casos diagnosticados por sexo, no período de 1972 a 2004.

De fato, estudos relevam que o hábito de fumar está associado a $45 \%$ das mortes por doença coronariana, $85 \%$ das mortes por doenças respiratórias obstrutivas crônicas, $25 \%$ das mortes por doença cérebro-vascular e $30 \%$ das mortes por câncer (ESTADOS UNIDOS, 1988; DOLL et al., 1994). É interessante que, embora o consumo de cigarros venha caindo na maioria dos países desenvolvidos, seu consumo global aumentou cerca de 50\% de 1975 a 1996 (WORLD BANK, 1993). Isto pode ser observado especialmente na Ásia, onde, não por mera coincidência, situam-se os países com as maiores incidências de câncer bucal do globo, como a Índia e o Paquistão.

Outro ponto que também tem de ser levado em consideração ao se traçar um perfil dos pacientes acometidos pelo câncer bucal, assim como por qualquer outra neoplasia maligna, é a raça, pois sempre existe a possibilidade de haver uma maior predisposição de uma em relação à outra. Dos 224 casos analisados neste levantamento, $74,6 \%$ acometeram indivíduos da raça branca, número semelhante aos encontrados por vários autores (CAMARINI, 1999; ABDO, 2001; SOUZA et al., 1996). Todavia, esta predileção por indivíduos de pele clara parece refletir muito mais uma tendência populacional do que se relacionar com a doença em si, porém somente com os avanços da biologia molecular é que poderse-á afirmar se a raça é, ou não, um fator de risco.

Agora, se essa dúvida paira em relação à raça, o mesmo não ocorre quanto à idade dos pacientes. Afinal, é do conhecimento de todos que à medida que se envelhece crescem as chances se desenvolver alguns tipos de câncer, entre eles o câncer bucal, o que se deve a um maior tempo de exposição aos fatores ambientais, associado a uma redução da capacidade regenerativa das células. Isto, talvez justifique o fato de que $86,2 \%$ dos casos analisados estavam entre a quinta e oitava década de vida, fazendo com que a média de idade dos pacientes no momento do diagnóstico fosse superior a 59 anos, valor muito próximo aos encontrados na literatura (ABDO, 2001; ANJOS HORA et al., 2003; GERVÁSIO et al., 2001) (Gráfico 3).

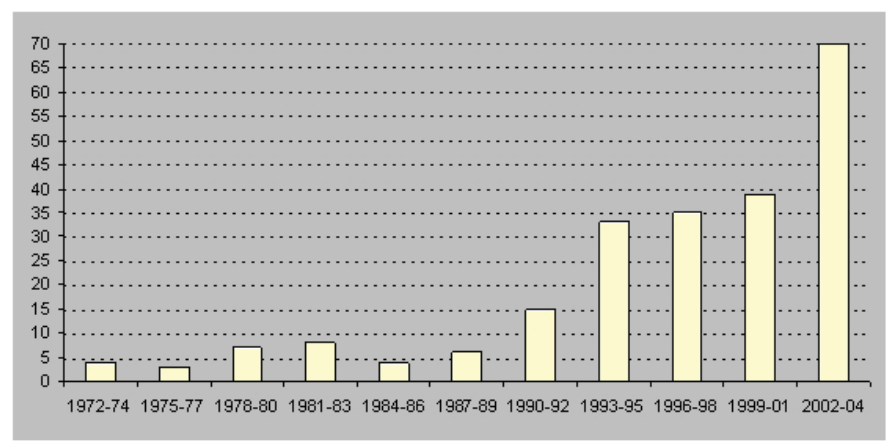

Gráfico 3. Distribuição do número de casos diagnosticados por faixa etária (I.N.E. - informação não-especificada). 
Embora a localização da lesão primária não seja considerada um dado epidemiológico, vale a pena discutir um pouco a seu respeito, chamando a atenção para as regiões que merecem um cuidado especial durante a realização do exame clínico. Segundo a literatura, essas regiões são: o lábio inferior, a borda lateral da língua, o soalho bucal e a área retromolar, sendo que essa ordem pode variar de um autor para outro (CAMARINI, 1999; ABDO, 2001; ANJOS HORA et al., 2003). Neste estudo, observou-se que a língua foi a região mais acometida, com $22,8 \%$ dos casos, seguida pelo lábio, $19,7 \%$ e soalho bucal, $14,3 \%$ (Gráfico 4). Todavia, muito pouco se sabe sobre o porquê destes locais apresentarem uma maior prevalência.

Frente a esses dados, pode-se afirmar, portanto, que o perfil dos casos de carcinoma epidermoide em mucosa bucal diagnosticados em São José dos Campos, SP, entre 1972 e 2004, está de acordo com o descrito na literatura, mostrando que o padrão epidemiológico apresentado pelo câncer bucal nesta cidade não difere daquele observado no restante do Brasil.

\section{Conclusão}

A elevada incidência do câncer bucal no Brasil o torna um problema de saúde pública. Contudo, a prevenção e o diagnóstico precoce ainda são as melhores armas das quais se dispõem para mudar este quadro. Assim, conhecer o perfil dos pacientes acometidos pelo câncer bucal é um passo importante para que os profissionais de saúde possam identificar de antemão aqueles indivíduos que apresentam um maior risco de desenvolver a doença, Ihes direcionado uma atenção especial quando da elaboração de programas de combate ao câncer.

\section{Referências}

ABDO E. N. Perfil do Paciente Portador do Carcinoma Epidermóide da Cavidade Bucal, em Tratamento num Hospital Vinculado ao Sistema Único de Saúde em Belo Horizonte. 2001. 99f. Dissertação (Mestrado) - Faculdade de Odontologia, Universidade Federal de Minas Gerais, Belo Horizonte, 2001.

ANJOS HORA, I. A. et al. Estudo Epidemiológico do Carcinoma Epidermóide de Boca no Estado de Sergipe. Ci. Odontol. Bras., São José dos Campos, v. 6, n. 2, p. 41-48, abr./jun., 2003.

BOYLE, P. et al. Epidemiology of Mouth Cancer in 1989: a Review. J. R. Soc. Med., London, v. 83, n. 11, p. 724-30, Nov. 1990.

BRASIL. Ministério da Saúde. Instituto Nacional do Câncer. Estimativa da Incidência e Mortalidade por Câncer no Brasil. Rio de Janeiro: INCA, 2002.

BRASIL. Ministério da Saúde. O Câncer de Boca no Brasil. Brasília: Ministério da Saúde. Boletim do Pro-Onco, Programa de Oncologia de Combate ao Câncer, Campanha Nacional de Combate ao Câncer, 1996.

BRASIL. Ministério da Saúde. Secretária de Atenção à Saúde. Instituto Nacional do Câncer. Coordenação de Prevenção e Vigilância. Estimativa 2005: Incidência de Câncer no Brasil. Rio de Janeiro: INCA, p. 94, 2004.

CAMARINI, E. T. Estudo Epidemiológico dos Carcinomas Espinocelulares de Boca dos Pacientes Atendidos nas Cidades de Bauru e Jaú, Estado de São Paulo, Brasil. 1999. 124p. Dissertação (Mestrado) - Faculdade de Odontologia de Bauru, Universidade de São Paulo, Bauru, 1999.
DOLL, R. et al. Mortality in Relation to Smoking: 40 Years' Observations on Male British Doctors. BMJ, London, v. 309, n. 6959, p. 901-911, Oct. 1994.

ESTADOS UNIDOS. Department of Health and Human Services. Office on Smoking and Health. The Health Consequences of Smoking: Nicotine Addiction: a Report of the Surgeon General. Rockville, Maryland, p. 639. 1988.

FIGUEIREDO, A. L. et al. Freqüência de Óbito por Câncer Bucal em Pernambuco no Período de 1979 a 1995. Rev. Cons. Reg. Odontol. Pernambuco, Recife, v. 3, n. 1, p. 39-43, jan./jun. 2000.

FRANCO, E. L. et al. Risk Factors for Oral Cancer in Brazil: a Casecontrol Study. Int. J. Cancer, New York, v. 43, n.6, p. 992-1000, June 1989.

GERVÁSIO, O. L. et al. Oral Squamous Cell Carcinoma: a Retrospective Study of 740 Cases in a Brazilian Population. Braz. Dent. J., Ribeirão Preto, v. 12, n. 1, p. 57-61, Jan./June 2001.

KO, Y. C. et al. Betel Quid Chewing, Cigarette Smoking and Alcohol Consumption Related to Oral Cancer in Taiwan. J. Oral Pathol. Med., Copenhagen, v. 24, n. 10, p. 450-453, Nov. 1995.

MÖLLER, H. Changing Incidence of Cancer of the Tongue, Oral Cavity, and Pharynx in Denmark. J. Oral Pathol. Med., Copenhagen, v. 18, n. 4, p. 224-229, Apr. 1989.

NEVILLE, B. W. et al. Oral \& Maxillofacial Pathology. Philadelphia: W. B. Saunders, p. 705, 1995.

PARISE JUNIOR, O. Câncer de Boca: Aspectos Básicos e Terapêuticos. São Paulo: Sarvier, p. 256, 2000.

SOUZA, A. et al. Epidemiologia do Carcinoma Epidermóide em Mucosa Bucal: Contribuição ao Estudo sobre Três Variáveis: Sexo, Faixa Etária e Raça. Rev. Odontol. UNICID, São Paulo, v. 8, n. 2, p. 127-134, jul./dez. 1996.

VIGNESWARAN, N. et al. Tobacco Use and Cancer. A Reappraisal. Oral Surg. Oral Med. Oral Pathol. Oral Radiol. Endod., St. Louis, v. 80, n. 2, p. 178-182, Aug. 1995.

WORLD BANK. Curbing the Epidemic: Governments and the Economics of Tobacco Control. Washington, DC, p.122, 1993. 